\title{
Maternal Periodontal Disease is Associated with Oxidative Stress during Pregnancy
}

\author{
M. Ashley Hickman, M.D. ${ }^{1}$, Kim A. Boggess, M.D. ${ }^{1}$, Kevin L. Moss ${ }^{2}$, James D. Beck, Ph.D. ${ }^{2}$, \\ and Steven Offenbacher, D.D.S., Ph.D. ${ }^{2}$ \\ ${ }^{1}$ University of North Carolina at Chapel Hill \\ ${ }^{2}$ University of North Carolina at Chapel Hill School of Dentistry, Chapel Hill, North Carolina
}

\begin{abstract}
We sought to determine if maternal periodontal disease is associated with oxidative stress as measured by serum 8 -isoprostane. A secondary analysis was conducted using prospective data from the Oral Conditions and Pregnancy Study. Healthy women enrolled at <26 weeks' gestational age underwent oral examination and serum sampling. Maternal periodontal disease status was categorized as healthy, mild, or moderate to severe by clinical criteria. Maternal serum was analyzed for 8-isoprostane using ultrasensitive enzyme-linked immunosorbent assay. Elevated 8-isoprostane level was defined as 275 th percentile. Maternal factors associated with elevated 8isoprostane were determined using chi-square or $t$ test. Multivariable logistic regression was used to assess association between elevated 8-isoprostane and maternal factors. Seven hundred ninetyone women had complete data. Median (interquartile) 8-isoprostane serum level was 1806 (16 to $81,870) \mathrm{pg} / \mathrm{dL}$. Using bivariate analysis, maternal age, race, marital status, utilization of public assistance, and mild or moderate to severe periodontal disease were associated with elevated serum 8-isoprostane. Using logistic regression, moderate to severe periodontal disease (adjusted odds ratio $2.9,95 \%$ confidence interval: 1.7 to 5.0 ) remained significantly associated with an elevated serum 8-isoprostane level. Maternal periodontal disease is associated with oxidative stress during pregnancy. Further study is needed to determine the role of maternal oxidative stress in periodontal disease-associated adverse pregnancy outcomes.
\end{abstract}

\section{Keywords}

Periodontal disease; oxidative stress; pregnancy; 8-isoprostane; biomarker

Oxidative stress is characterized by a state of imbalance between free radicals and antioxidant defenses. Free radicals are produced endogenously as part of the oxidative burst of phagocyte activation, and they are encountered via exogenous production from sources such as cigarette smoke. When an excess production of reactive oxygen species is formed, oxidative stress ensues. A member of the isoprostane family, 8-isoprostane $\mathrm{PGF}_{2 a}$ (8-iso),

Copyright (C) 2011 by Thieme Medical Publishers, Inc.

Address for correspondence and reprint requests: M. Ashley Hickman, M.D., Fellow and Clinical Instructor, Division of Maternal Fetal Medicine, Department of Obstetrics and Gynecology, University of North Carolina School of Medicine, Chapel Hill, 3010 Old Clinic Bldg. CB \#7516, Chapel Hill, NC 27599-7516 (m.ashley.hickman@gmail.com). 
has been advocated as the "gold standard" for measurement of in vivo "whole-body" oxidative stress. ${ }^{1,2}$ The 8 -iso is predominantly generated by cyclo-oxygenase-independent free radical attack on arachidonic acid in cell membranes.

Oxidative stress plays an important role in the pathogenesis of periodontal disease and its complications. ${ }^{3,4}$ Individuals with periodontal disease display high levels of local and systemic biomarkers of oxidative stress. ${ }^{5}$ This has potential relevance for the pregnant woman, as periodontal disease affects 5 to $40 \%$ of pregnant women. Additionally, periodontal disease has been associated with preeclampsia and adverse pregnancy outcomes characterized by impaired antioxidant defense and maternal oxidative stress. ${ }^{6-8}$ Women destined to develop preeclampsia have increased systemic concentrations of 8-iso early in pregnancy, prior to the clinical manifestation of the disease. ${ }^{9}$ Recently, Wikström and colleagues demonstrated an association between elevated placental 8-iso and early onset preeclampsia. ${ }^{10}$ Maternal periodontal disease may be a stimulus for this increase in oxidative stress.

The objective of this analysis was to determine if maternal periodontal disease was associated with oxidative stress during pregnancy, which may suggest a possible therapeutic target to reduce periodontal disease-associated pregnancy complications.

\section{METHODS}

This is a secondary analysis of data from the Oral Conditions and Pregnancy study, which was a prospective cohort performed at the Center for Oral and Systemic Disease, School of Dentistry, University of North Carolina, Chapel Hill, North Carolina, in collaboration with Duke University Medical Center, Durham, North Carolina, from December 1997 through July 2001. The primary goal was to evaluate obstetric outcome in women with various stages of periodontal health and disease. Study design and procedures have been fully described previously. ${ }^{11}$ The Duke University Medical Center and the University of North Carolina Institutional Review Boards granted approval to conduct this study, and written informed consent was obtained from all study participants.

Over a 42-month period, healthy women with a singleton pregnancy were enrolled at $<26$ weeks of gestation, with $40 \%$ of the women enrolled at less than 14 weeks' gestation. Demographic information, health behaviors, and medical history data were obtained by subject questionnaire and direct interview at the first visit, which were reviewed by a physician at the first prenatal visit. Race was determined by maternal self-report. Women were considered to have used alcohol or tobacco if, by their report, they had used any since their last missed menstrual period. All women underwent periodontal examinations and maternal serum specimen collection at enrollment. Periodontal disease status was defined as "healthy," "mild," or "moderate to severe" based on clinical criteria. ${ }^{6}$ For the purpose of this analysis, periodontal health was defined as the absence of gingival pocket depths greater than or equal to $4 \mathrm{~mm}$ and no pockets greater than $3 \mathrm{~mm}$ that bled on probing. Mild periodontal disease was defined as one or more tooth sites with greater than or equal to 4$\mathrm{mm}$ pocket depth or one or more pockets that bled on probing, up to 15 tooth sites. Moderate 
to severe periodontal disease was considered present if 15 or more tooth sites had pocket depth greater than or equal to $4 \mathrm{~mm} .{ }^{11}$

Blood was drawn from all women at the time of enrollment via standard venipuncture technique. Samples were then centrifuged and frozen at $-80^{\circ} \mathrm{C}$. For this analysis, total serum 8-iso quantification was performed using a commercially available ultrasensitive enzymelinked immunoassay (Assay Designs' Direct 8-iso-PGF $2 \mathrm{a}$ kit, Biomol, Hamburg, Germany) at a single laboratory at the Center for Oral and Systemic Disease, School of Dentistry, University of North Carolina, Chapel Hill, North Carolina. The lower limit of detection of this assay is of $0.022 \mathrm{pg} / \mathrm{mL}$, with inter-and intra-assay variability of $5.4 \%$ and $10.4 \%$, respectively. There are no currently accepted standard normal values for 8-iso, and the literature does not suggest a threshold value above which clinically relevant findings are seen. Therefore, for this analysis, we defined elevated as serum quartile levels of 8-iso greater than or equal to the 75 th percentile, which corresponded to a level $>81,870 \mathrm{pg} / \mathrm{mL}$ in our cohort. Bivariate analysis was performed to determine association between elevated 8iso and maternal factors using chi-square or $t$ test. Maternal factors that were associated with an elevated 8-iso level at $p<0.1$ were entered into a multivariable logistic regression model to determine the adjusted odds ratios and $95 \%$ confidence intervals (CIs) for an elevated 8iso level. A more parsimonious model was then determined by backward, stepwise logistic regression. Statistical analyses were performed using Statistical Analysis Software, version 9.1 (SAS, Cary, NC).

\section{RESULTS}

A total of 791 women had complete data and serum samples available for analysis. The median gestational age at enrollment was 14 weeks with a range of 4 to 26 weeks. Demographic, medical, and obstetric data of the women who made up the cohort for this analysis are shown in Table 1. Maternal age, race, marital status, low socioeconomic status as indicated by eligibility for the Women and Infant Care program and/or food stamps, and presence of moderate to severe periodontal disease, were associated with elevated serum 8iso level.

Multivariable regression models for an elevated serum 8-iso level are shown in Tables 2 and 3. After accounting for potential confounders such as socioeconomic status, maternal moderate to severe periodontal disease in early pregnancy remained significantly associated with an elevated serum 8-iso level (Table 3).

\section{DISCUSSION}

We found that low socioeconomic status and the presence of moderate to severe periodontal disease are significantly associated with increased oxidative stress as measured by maternal serum 8-iso. Pregnancy is inherently a state of oxidative stress due to the increased metabolic activity in placental mitochondria and the reduced scavenging power of antioxidants. The isoprostanes are a specific marker for oxidative damage to lipids from endogenous lipid peroxidation. They are prostaglandin-like compounds derived by autooxidation of the arachidonic acid moiety. ${ }^{12}$ The F2 isoprostanes, including 8 -iso, are 
chemically stable, specific products of peroxidation. They are formed in vivo and are present in detectable amounts in all normal tissues and biological fluids. In addition to serving as a marker of oxidative damage, they possess biological activity of their own via vasoconstrictive effects in the lung and kidney. The F2 isoprostanes do not exhibit normal diurnal variation, and they are known to substantially increase in animal models of oxidant injury, without influence of the lipid content of the diet. ${ }^{13}$ In vitro measurement of the F2 isoprostanes is considered the best available marker of in vivo oxidative stress.

Periodontal disease is a significant oral health condition commonly encountered in pregnant women. ${ }^{11}$ The association between periodontal disease and adverse pregnancy outcome has been the subject of several studies with inconsistent conclusions. Some have demonstrated an association between periodontal disease and adverse pregnancy outcomes such as preterm birth, preeclampsia, and low birth weight in various populations, ${ }^{14-17}$ although others have not confirmed this association. ${ }^{18}$ Perhaps this disparity is due to differences in the definitions used for assessing the presence of periodontal disease. ${ }^{19}$ However, in our cohort of women, maternal periodontal disease has been associated with elevated maternal inflammation, ${ }^{20}$ which may contribute to the risk for preeclampsia. ${ }^{21}$ Others have also noted an association between elevated levels of oxidative stress in women with preeclampsia compared with gestation-matched control women. ${ }^{10}$ Data suggest that the increased 8-iso could be due to increased oxidative stress in preeclampsia or a decrease in renal clearance with the vasoconstrictive activity of 8-iso a potential contributor to the pathogenesis of preeclampsia. ${ }^{22}$ Our data demonstrate that moderate to severe periodontal disease is associated with maternal oxidative stress as measured by serum 8 -iso, supporting the findings of others that the effects of periodontal disease do not remain localized.

Prior studies examining the impact of localized treatment of periodontal disease during pregnancy to decrease adverse outcome have had mixed results. In their large intervention trial, Offenbacher et al found a significant reduction in preterm birth among women who received treatment of their periodontal disease during pregnancy. ${ }^{23}$ However, most recently, Macones et al reported the results of their randomized clinical trial of periodontal disease to reduce preterm birth. Notably, the overall occurrence of preterm birth was not decreased in the women who received treatment; however, among women who received treatment, a greater proportion experienced a medically indicated preterm delivery (3.3\% versus $1.1 \%$; relative risk 3.01 ; $95 \% \mathrm{CI}, 0.95$ to 4.24$).{ }^{24}$ The next step is to determine if maternal oxidative stress in response to periodontal disease may be part of the pathophysiology of periodontal-associated adverse pregnancy outcomes and whether the inclusion of antioxidant therapy with periodontal disease treatment might improve obstetric outcome in affected women.

Several limitations of our study merit discussion. Our study population included a significant number of African-American women who are known to have worse periodontal disease than their Caucasian counterparts and have higher concentrations of systemic inflammation. ${ }^{20,25}$ Racial, educational, and socioeconomic status disparities have been previously associated with periodontal disease. African-Americans, especially those of lower socioeconomic status, are less likely to seek preventive dental services, and they have higher rates of periodontal disease compared with non-Hispanic white populations and 
African-Americans of higher socioeconomic status. ${ }^{25}$ Internationally, the reports of increased periodontal disease among members of lower socioeconomic groups are noted even in the setting of universal, government-sponsored dental care, which points to the significant effects education and attitude toward dental health may have. ${ }^{26,27}$

In conclusion, moderate to severe periodontal disease is associated with oxidative stress in early pregnancy, as measured by serum 8 -iso. Future research is needed to gather longitudinal data on biomarkers of oxidative stress, to determine if the treatment of maternal periodontal disease alters maternal oxidative stress during pregnancy and whether antioxidant therapy should be considered as part of the treatment of periodontal disease during pregnancy.

\section{Acknowledgments}

This study was supported by NIDCR grant RO-1-DE-12453.

\section{References}

1. Chapple IL. Reactive oxygen species and antioxidants in inflammatory diseases. J Clin Periodontol. 1997; 24:287-296. [PubMed: 9178107]

2. Milne GL, Yin H, Brooks JD, Sanchez S, Jackson Roberts LII, Morrow JD. Quantification of F2isoprostanes in biological fluids and tissues as a measure of oxidant stress. Methods Enzymol. 2007; 433:113-126. [PubMed: 17954231]

3. Borges I Jr, Moreira EA, Filho DW, de Oliveira TB, da Silva MB, Fröde TS. Proinflammatory and oxidative stress markers in patients with periodontal disease. Mediators Inflamm. 2007; 2007:45794. [PubMed: 18288271]

4. Panjamurthy K, Manoharan S, Ramachandran CR. Lipid peroxidation and antioxidant status in patients with periodontitis. Cell Mol Biol Lett. 2005; 10:255-264. [PubMed: 16010291]

5. Chapple IL, Matthews JB. The role of reactive oxygen and antioxidant species in periodontal tissue destruction. Periodontol 2000. 2007; 43:160-232. [PubMed: 17214840]

6. Boggess KA, Lieff S, Murtha AP, Moss K, Beck J, Offenbacher S. Maternal periodontal disease is associated with an increased risk for preeclampsia. Obstet Gynecol. 2003; 101:227-231. [PubMed: 12576243]

7. Uotila J, Tuimala R, Pyykkö K, Ahotupa M. Pregnancy-induced hypertension is associated with changes in maternal and umbilical blood antioxidants. Gynecol Obstet Invest. 1993; 36:153-157. [PubMed: 8244188]

8. Hubel CA. Oxidative stress in the pathogenesis of pree-clampsia. Proc Soc Exp Biol Med. 1999; 222:222-235. [PubMed: 10601881]

9. Chappell LC, Seed PT, Briley A, et al. A longitudinal study of biochemical variables in women at risk of preeclampsia. Am J Obstet Gynecol. 2002; 187:127-136. [PubMed: 12114900]

10. Wikström AK, Nash P, Eriksson UJ, Olovsson MH. Evidence of increased oxidative stress and a change in the plasminogen activator inhibitor (PAI)-1 to PAI-2 ratio in early-onset but not lateonset preeclampsia. Am J Obstet Gynecol. 2009; 201:597, e1-e8. [PubMed: 19683696]

11. Lieff S, Boggess KA, Murtha AP, et al. The oral conditions and pregnancy study: periodontal status of a cohort of pregnant women. J Periodontol. 2004; 75:116-126. [PubMed: 15025223]

12. Morrow JD, Harris TM, Roberts LJ II. Noncyclooxygenase oxidative formation of a series of novel prostaglandins: analytical ramifications for measurement of eicosanoids. Anal Biochem. 1990; 184:1-10. [PubMed: 2321745]

13. Montuschi P, Barnes PJ, Roberts LJ II. Isoprostanes: markers and mediators of oxidative stress. FASEB J. 2004; 18:1791-1800. [PubMed: 15576482]

14. Xiong X, Buekens P, Fraser WD, Beck J, Offenbacher S. Periodontal disease and adverse pregnancy outcomes: a systematic review. BJOG. 2006; 113:135-143. [PubMed: 16411989] 
15. Shub A, Swain JR, Newnham JP. Periodontal disease and adverse pregnancy outcomes. J Matern Fetal Neonatal Med. 2006; 19:521-528. [PubMed: 16966119]

16. Siqueira FM, Cota LO, Costa JE, Haddad JP, Lana AM, Costa FO. Maternal periodontitis as a potential risk variable for preeclampsia: a case-control study. J Periodontol. 2008; 79:207-215. [PubMed: 18251635]

17. Shub A, Wong C, Jennings B, Swain JR, Newnham JP. Maternal periodontal disease and perinatal mortality. Aust N Z J Obstet Gynaecol. 2009; 49:130-136. [PubMed: 19441161]

18. Srinivas SK, Sammel MD, Stamilio DM, et al. Periodontal disease and adverse pregnancy outcomes: is there an association? Am J Obstet Gynecol. 2009; 200:497, e1-e8. [PubMed: 19375568]

19. Manau C, Echeverria A, Agueda A, Guerrero A, Echeverria JJ. Periodontal disease definition may determine the association between periodontitis and pregnancy outcomes. J Clin Periodontol. 2008; 35:385-397. [PubMed: 18341599]

20. Horton AL, Boggess KA, Moss KL, Jared HL, Beck J, Offenbacher S. Periodontal disease early in pregnancy is associated with maternal systemic inflammation among African American women. $\mathrm{J}$ Periodontol. 2008; 79:1127-1132. [PubMed: 18597593]

21. Ruma M, Boggess K, Moss K, et al. Maternal periodontal disease, systemic inflammation, and risk for preeclampsia. Am J Obstet Gynecol. 2008; 198:389, e1-e5. [PubMed: 18295179]

22. Barden A, Beilin LJ, Ritchie J, Croft KD, Walters BN, Michael CA. Plasma and urinary 8isoprostane as an indicator of lipid peroxidation in pre-eclampsia and normal pregnancy. Clin Sci (Lond). 1996; 91:711-718. [PubMed: 8976806]

23. Offenbacher S, Beck JD, Jared HL, et al. Maternal Oral Therapy to Reduce Obstetric Risk (MOTOR) Investigators. Effects of periodontal therapy on rate of preterm delivery: a randomized controlled trial. Obstet Gynecol. 2009; 114:551-559. [PubMed: 19701034]

24. Macones GA, Parry S, Nelson DB, et al. Treatment of localized periodontal disease in pregnancy does not reduce the occurrence of preterm birth: results from the Periodontal Infections and Prematurity Study (PIPS). Am J Obstet Gynecol. 2010; 202:147, e1-e8. [PubMed: 20113691]

25. Gilbert GH, Duncan RP, Shelton BJ. Social determinants of tooth loss. Health Serv Res. 2003; 38(6 Pt 2):1843-1862. [PubMed: 14727800]

26. Christensen LB, Petersen PE, Steding-Jessen M. Consumption of dental services among adults in Denmark 1994-2003. Eur J Oral Sci. 2007; 115:174-179. [PubMed: 17587291]

27. Wamala S, Merlo J, Boström G. Inequity in access to dental care services explains current socioeconomic disparities in oral health: the Swedish National Surveys of Public Health 20042005. J Epidemiol Community Health. 2006; 60:1027-1033. [PubMed: 17108297] 
Table 1

Characteristics of the Study Cohort

\begin{tabular}{|c|c|c|c|c|}
\hline Maternal Characteristics & $\begin{array}{l}\text { Whole Cohort }(n \\
=791)\end{array}$ & $\begin{array}{l}\text { Serum 8-Isoprostane } \\
<75 \text { th Percentile }(n=593)\end{array}$ & $\begin{array}{l}\text { Serum 8-Isoprostane } \\
\geq 75 \text { th Percentile }(n=198)\end{array}$ & $p$ Value \\
\hline Age, y $($ mean \pm SD) & $28 \pm 6.5$ & $29 \pm 6.5$ & $27.0 \pm 6.6$ & 0.0007 \\
\hline Weight at enrollment (pounds, mean $\pm \mathrm{SD}$ ) & $162 \pm 44.7$ & $162 \pm 42.5$ & $167 \pm 51.2$ & 0.08 \\
\hline Race & & & & $<0.0001$ \\
\hline Caucasian & $367(46)$ & $311(52)$ & $68(34)$ & \\
\hline African-American & $379(48)$ & $251(42)$ & $116(59)$ & \\
\hline Other & $45(6)$ & $31(5)$ & $14(7)$ & \\
\hline Married & $402(51)$ & $324(55)$ & $78(39)$ & 0.0002 \\
\hline Unmarried & $389(49)$ & $269(69)$ & $120(61)$ & \\
\hline WIC/food stamps & $174(22)$ & $104(60)$ & $70(40)$ & $<0.0001$ \\
\hline First pregnancy & $313(40)$ & $236(40)$ & $77(39)$ & NS \\
\hline Tobacco use & $135(17)$ & $93(16)$ & $44(22)$ & 0.07 \\
\hline Alcohol use & $123(16)$ & $88(15)$ & $35(18)$ & NS \\
\hline Periodontal disease status & & & & $<0.0001$ \\
\hline Healthy & $218(28)$ & $182(31)$ & $36(18)$ & \\
\hline Mild & $459(58)$ & 347 (59) & $112(57)$ & \\
\hline Moderate-severe & $114(41)$ & $64(11)$ & $50(25)$ & \\
\hline
\end{tabular}

Data presented as $n(\%)$ unless otherwise noted. SD, standard deviation; WIC, Women and Infant Care program. 
Table 2

Full Multivariable Regression Model for an Elevated Serum 8-Isoprostane Level

\begin{tabular}{ll}
\hline Maternal Characteristic & Adjusted Odds Ratio ${ }^{*}$ (95\% Confidence Interval) \\
\hline Age & $0.98(0.95-1.01)$ \\
Weight & $1.0(1.00-1.01)$ \\
African-American race & $1.18(0.78-1.79)$ \\
Married & $0.94(0.61-1.45)$ \\
WIC/food stamp eligible & $1.74(1.16-2.61)$ \\
Smoked during pregnancy & $1.10(0.71-1.71)$ \\
Mild periodontal disease & $1.38(0.82-2.00)$ \\
Moderate/severe periodontal disease & $2.59(1.45-4.60)$ \\
\hline
\end{tabular}

* Adjusted for all variables listed.

WIC, Women and Infant Care program 
Table 3

Reduced Multivariable Regression Model for an Elevated Serum 8-Isoprostane Level

\begin{tabular}{ll}
\hline Maternal Characteristic & Adjusted Odds Ratio* (95\% CI) \\
\hline Age & $0.97(0.95-0.99)$ \\
WIC/food stamp eligible & $1.87(1.26-2.77)$ \\
Mild periodontal disease & $1.37(0.89-2.12)$ \\
Moderate-severe periodontal disease & $2.89(1.67-5.00)$ \\
\hline * Adjusted for all variables listed. &
\end{tabular}

CI, confidence interval; WIC, Women and Infant Care program. 